items were recorded. This trend appeared more marked specifically looking at GP referrals (20\% vs $46 \%$ ).

Conclusions This analysis of referrals would suggest that the quality of referral letters is linked to clinic outcome. Referrals containing very little information resulted in more patients being discharged directly from the clinic without investigation. Referral guidelines for general practice will hopefully improve the quality of referrals.

\section{P251 PREVALENCE AND TREATMENT OUTCOME OF OBSTRUCTIVE SLEEP APNOEA (OSA) DIAGNOSED FOLLOWING PREOPERATIVE SCREENING COMPARED WITH GP OR OTHER CLINICIAN REFERRAL}

A Dwarakanath, V Palissery, MW Elliott; St. James University Hospital, Leeds, United Kingdom

\subsection{6/thoraxjnl-2013-204457.403}

Introduction OSA is very prevalent and has potential implications perioperatively. Preoperative screening may identify high risk patients and treatment with CPAP may reduce perioperative complications, though this is unproven. We evaluated the treatment outcome and long term compliance with CPAP in patients diagnosed through preoperative screening and compared it with patients diagnosed with OSAS following GP or other clinician referral.

Method Over 2 years (October 2009-2011) 1412 patients (males-62\%) had sleep studies (oximetry or respiratory variable). $44 \%$ were referred from the preassessment clinic following screening for possible OSA. The prevalence of sleep disordered breathing, the Epworth Sleepiness Score (ESS) and among those referred for a CPAP trial the outcome, long-term compliance and average use per night were compared between preassessment and clinician referred patients.

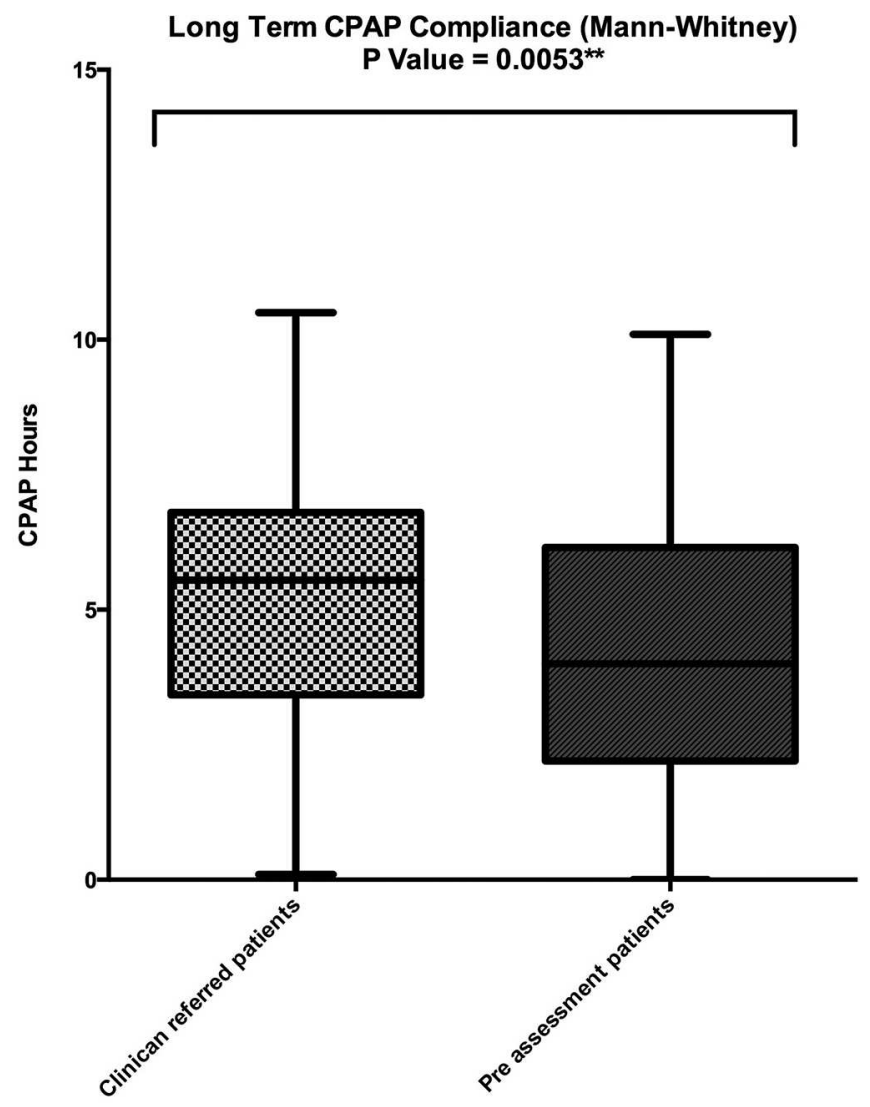

Abstract P251 Figure 1.
Results The prevalence of OSA was $62 \%$ and $58 \%$ in the clinician referred and preassessment patients respectively. There was a significant difference in age $(61+/-16 \quad \mathrm{v} / \mathrm{s} \quad 55 \quad+/-13$, $\mathrm{P}=<0.0001)$ and ESS $(11+/-6 \mathrm{v} / \mathrm{s} 8+/-5, \mathrm{P}=<0.0001)$ between the two groups. Clinician referred patients were more likely to be commenced on CPAP ( $\mathrm{P}=<0.0001$, OR- 2.79). Preassessment patients with mild OSA who were prescribed CPAP were more likely to fail the CPAP trial $(\mathrm{P}=0.01$, OR3.02 ) and were less likely to continue CPAP treatment after one year $(\mathrm{P}=0.02$, OR-2.1). No difference was seen between the groups in patients with moderate or severe OSA. There was a significant difference in the median CPAP usage, 5.5 hours v/s 4 hours (Mann Whitney, $\mathrm{P}=0.0053$, figure-1). Both groups reported a significant improvement in ESS with CPAP ( $\Delta$ ESS-5 and $\Delta$ ESS-4, $\mathrm{P}=<0.0001$ ) between the clinician referred and preassessment patients respectively.

Conclusions The prevalence of OSA was similar in patients referred following preoperative screening or from another clinician, but preassessment patients were younger and less symptomatic. There was no difference in short or long term CPAP use in patients with moderate or severe OSA, but preassessment patients with mild OSA were less likely to use CPAP in the short or longer term. Opportunistic screening of patients awaiting surgery is worthwhile, independently of any effect of CPAP upon surgical outcomes.

\section{P252 RESIDUAL DROWSINESS AND CPAP COMPLIANCE IN OSAS PATIENTS AND THE DVLA- ON BEHALF OF THE BRITISH THORACIC SOCIETY SLEEP APNOEA SAG}

${ }^{1}$ A Dwarakanath, ${ }^{1} \mathrm{D}$ Ghosh, ${ }^{2} \mathrm{SL}$ Jamson, ${ }^{3} \mathrm{PD}$ Baxter, ${ }^{4} \mathrm{M}$ Twiddy, ${ }^{1} \mathrm{MW}$ Elliott; ${ }^{1}$ St. James University Hospital, Leeds, United Kingdom; ${ }^{2}$ Institute for Transport Studies, University of Leeds, Leeds, United Kingdom; ${ }^{3}$ Division of Biostatistics, LIGHT, Centre for Epidemiology and Biostatistics, University of Leeds, Leeds, United Kingdom; ${ }^{4}$ Leeds Institute of Health Sciences, Leeds, United Kingdom

\subsection{6/thoraxjnl-2013-204457.404}

Introduction Clinicians are often asked to complete forms about patients with OSAS by the DVLA. We evaluated the current practice of assessing residual drowsiness, CPAP compliance and whether objective testing is undertaken by clinicians to assess an individual's fitness for driving.

Methods Clinicians who complete the DVLA medical forms (SL1 and SL1V) were invited to participate in a web-based survey. Respondents were presented with five vignettes of patients with OSAS offered CPAP and to answer the questions posed by the DVLA about residual drowsiness ("excessive" (SL1) or

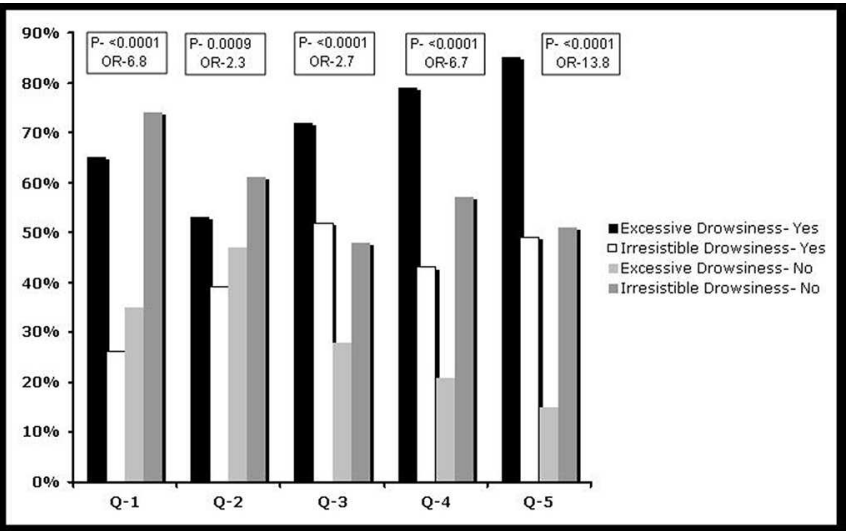

Abstract P252 Figure 1. 
"irresistible" (SL1V)) and adequacy of CPAP compliance. They were also asked about their use of objective tests.

Results 178 respondents completed the survey. There was poor agreement among clinicians regarding the presence of residual drowsiness (McNemar's test, figure-1). In response to the DVLA question about "excessive" drowsiness, the patient had a 1 in 2.57 (range-1.12 to 5.66), and about "irresistible" drowsiness, a 1 in 1.32 (range- 1.04 to 2.84), chance of being given a different answer depending on the clinician seen. Furthermore in each vignette the same clinician was more likely to say "yes" to "excessive" than to "irresistible" (71 +/-12\% v/s $42+/-10 \%$, P-0.0045). There was also a lack of consensus as to what constitutes “adequate CPAP compliance”. Across the vignettes there was minimum of 1 in 1.7 and a maximum of 1 in 6.7 chance of disagreement amongst the clinicians (median- 3 , range 1.7 to 6.7 ). $1 \%$ of clinicians always and $4 \%$ frequently use objective tests to help in their assessment. They are more likely to use in professional drivers as compared to non professional drivers $(52 \% \mathrm{v} / \mathrm{s} 38 \%$, P0.0002, OR-1.75). Tests used were MSLT (34\%), OSLER (29\%), MWT (28\%) and divided attention driving simulator (9\%).

Conclusions The information that the DVLA is given may vary markedly depending upon which clinician completes the form. Furthermore the same clinician may give a different impression to the DVLA depending on which form they are asked to complete. Objective testing is not undertaken routinely. Better guidance and better objective tests are needed to ensure consistency of the information that the DVLA is given.

\section{P253 OBSTRUCTIVE SLEEP APNOEA SYNDROME: PATIENTS' EXPERIENCE OF THE DRIVER AND VEHICLE LICENSING AGENCY}

M Mason, N Oscroft, M Davies, T Quinnell, I Smith; Respiratory Support and Sleep Centre, Papworth Hospital NHS Foundation Trust, Papworth Everard, Cambridge, UK

\subsection{6/thoraxjnl-2013-204457.405}

Introduction Driver's licence holders have a legal obligation to inform the Driver and Vehicle Licensing Authority (DVLA) if diagnosed with Obstructive Sleep Apnoea Syndrome (OSAS). This requirement may cause considerable anxiety but few data are available to advise patients on the likely outcomes following such notification.

Methods Patients diagnosed with OSAS and offered CPAP between $1^{\text {st }}$ October 2009 and $31^{\text {st }}$ March 2010 were surveyed

\begin{tabular}{ll} 
Abstract P253 Table 1. Characteristics of the patient population \\
sample \\
\hline Characteristic & \\
Mean Age years (range) & $54(31-82)$ \\
Gender (male/female) & $2.7: 1$ \\
BMI (kg/m $)$ & $38(9)$ \\
ESS at diagnosis & $13(5)$ \\
ESS post treatment & $7(5)$ \\
ODI at diagnosis & $21(20)$ \\
ODI post treatment & $4(12)$ \\
Compliance hours/night & $5.8(2)$ \\
(measured by CPAP device) & \\
Compliance with CPAP treatment $>3.5$ hours/night & $72 \%$ \\
Recorded evidence in medical notes of advice regarding the DVLA & $79 \%$ \\
\hline
\end{tabular}

BMI, body mass index; ESS, Epworth Sleepiness Score; ODI, oxygen desaturation index (4\% drop in saturation/hour); CPAP, continuous positive airway pressure

Data presented as mean (SD) unless specified anonymously and asked to report on: a) symptoms of sleepiness whilst driving before and after treatment; b) if they recalled being given advice about contacting the DVLA by healthcare professionals; c) whether they contacted the DVLA; d) the response of the DVLA if notified. The survey was performed between $1^{\text {st }}$ June and $31^{\text {st }}$ August 2010. To provide a description of the population surveyed $67(10 \%)$ case notes were chosen at random from the population surveyed, reviewed and descriptive data extracted.

Results Six hundred and seventy three patients were surveyed and 297 (44\%) responded. 92\% were category B licence holders. The data outlining the surveyed population are in Table 1.

Sixty percent and $16 \%$ respectively reported no and moderate to severe sleepiness whilst driving, prior to treatment. Two hundred and six patients (69\%) recalled being given advice about driving by a health care professional and of those 161 patients (78\%) had informed the DVLA of their diagnosis. In total 197 patients (66\%) had informed the DVLA of their OSAS. The DVLA asked 8\% (16/ 197) to stop driving temporarily of which $80 / 197$ contacted the DVLA prior to starting treatment. Five patients $(2.5 \%)$ were deemed not fit to drive by the DVLA in the long term. The mean (SD) time for the DVLA to reach a decision was 29 (33) days.

Conclusion In summary, recognising its limits, in particular the risk of responder bias, this survey shows most OSAS patients offered CPAP do not experience problems with driving licence retention if they contact the DVLA. The DVLA infrequently ask patients to stop driving. Approximately one third of patients had not informed the DVLA of their OSAS during the timeframe of this survey.

\section{P254 PREDICTING WHO NEEDS A HUMIDIFIER WITH CPAP}

L Thirugnanasothy, J Littlemore, J Oliver, P Close, SD West; Freeman Hospital, Newcastleupon-tyne, England

\subsection{6/thoraxjnl-2013-204457.406}

Introduction Some patients using continuous positive airway pressure (CPAP) for the treatment of Obstructive Sleep Apnoea (OSA) require the addition of a heated humidifier to their CPAP machine. This is used to combat symptoms of dry nose and mouth and blocked nose which may limit CPAP adherence. We sought to establish whether there are any patient predictors for whether a humidifier will subsequently be needed. This might allow provision of more economical integrated CPAP/humidifiers. Methods All patients commencing CPAP over a one year period completed a questionnaire with a member of the Sleep team at the time of CPAP set-up. The questionnaire asked about symptoms prior to CPAP including blocked, dry or runny nose and dry mouth on waking. Details of sleep study parameters such as ODI and AHI were obtained from hospital notes, along with anthropometric measures of body mass index (BMI) and neck size. The questionnaire also enquired about previous medical history (including ENT surgery), medications, smoking history and bedroom environment. Patients were given humidifiers according to usual practice as required after CPAP commencement, according to symptoms. The CPAP database was reviewed at the time of analysis to determine which patients had received humidifiers.

Results Questionnaires were completed by 185 people commencing CPAP from January 2012. The mean (SD) age of this group was 53 years (11.7), BMI $36.6 \mathrm{~kg} / \mathrm{m}^{2}(7.1)$, neck size $43.9 \mathrm{~cm}$ (4.4), ODI 29.3 (23). The proportions of different severities of OSA were $19 \%$ mild, $34 \%$ moderate and $47 \%$ severe. The frequencies of symptoms prior to CPAP were $87 \%$ dry mouth, $54 \%$ blocked nose, $40 \%$ dry nose and $22 \%$ runny nose. 